\title{
Inducible nitric oxide synthase mediates the change from retinal to vitreal neovascularization in ischemic retinopathy
}

\author{
Florian Sennlaub, ${ }^{1,2}$ Yves Courtois, ${ }^{1}$ and Olivier Goureau ${ }^{1}$ \\ ${ }^{1}$ Développement, Vieillissement, et Pathologie de la Rétine, Institut National de la Santé et de la Recherche Médicale \\ (INSERM) Unité 450, Association Claude Bernard, Paris, France \\ ${ }^{2}$ Augenklinik der Charité, Virchow-Klinikum, Humboldt Universität, Berlin, Germany \\ Address correspondence to: Florian Sennlaub, Développement, Vieillissement, et Pathologie de la Rétine, \\ Institut National de la Santé et de la Recherche Médicale Unité 450, 29, rue Wilhem, 75016 Paris, France. \\ Phone: 33145252193; Fax: 33140500195; E-mail: fsennlau@infobiogen.fr.
}

Received for publication July 26, 2000, and accepted in revised form January 31, 2001.

Intravitreal neovascular diseases are a major cause of blindness worldwide. It remains unclear why neovessels in many retinal diseases spread into the physiologically nonvascularized vitreous rather than into the ischemic retinal areas, where the angiogenic factors are released. Here we show that inducible nitric oxide synthase (iNOS) is expressed in the ischemic retina. Using iNOS knockout mice and the iNOS inhibitor $1400 \mathrm{~W}$, we demonstrate that iNOS expression inhibits angiogenesis locally in the avascular retina, mediated at least in part by a downregulation of VEGF receptor 2 (VEGFR2) in cells adjacent to iNOS-expressing cells. At the same time, pathological intravitreal neovascularization is considerably stronger in iNOS-expressing animals. These findings demonstrate that iNOS plays a crucial role in retinal neovascular disease and show that it offers an ideal target for the control of vitreal neovascularization through improvement of the vascularization of the hypoxic retina.

J. Clin. Invest. 107:717-725 (2001).

\section{Introduction}

Intravitreal neovascularization - e.g., diabetes mellitus, retinopathy of prematurity, or retinal vein occlusion is a major cause of blindness worldwide (1). Retinal ischemia is thought to be a common precursor to vitreal neovascularization in retinal diseases (2). Although the understanding of the mechanisms involved in the formation of new vessels has much improved in recent years, it remains unclear why neovascularization in ischemic retinopathy occurs mainly in the vitreous and not in the hypoxic retina where the angiogenic factors (notably VEGF) are secreted.

Nitric oxide (NO) is a free radical, produced from L-arginine by NO synthases (NOS) (3) and is involved in diverse processes such as neurotransmission, vasodilatation, host defense, and inflammation (4-6). The existence of at least three different forms of NOS, each encoded by a specific gene, has been demonstrated (3). Two of these isoforms are continuously expressed in the body: nNOS (NOS-I), essentially present in neurons of the central and peripheral nervous system; and eNOS (NOS-III), predominantly found in the plasmic membrane of vascular endothelial cells $(3,4)$. However, the third isoform, inducible iNOS (NOS-II), is expressed in different cell types only after transcriptional activation by endotoxins and cytokines (7). The NO produced by iNOS acts as a microbicidal and antiviral agent $(6,7)$ in immunological defenses. $\mathrm{NO}$ is also thought to be a mediator of autoimmune and inflammatory responses.
$\mathrm{NO}$ is known to influence neovascularization in a variety of models of angiogenesis, and its effect can be proangiogenic (8-10) or antiangiogenic (11-13). These seemingly contradictory data may be explained by the action of different NOS isoforms in the different models used. There is increasing evidence that eNOS plays a proangiogenic role $(14,15)$, and it seems that iNOS has an antiangiogenic effect $(16,17)$. The role of $\mathrm{nNOS}$ in angiogenesis remains undetermined.

To investigate the role of iNOS in retinal neovascularization, we studied the neovascular response of mice lacking iNOS in oxygen-induced proliferative retinopathy.

\section{Methods}

Animals. C57BL/6×129SvEv mice with a targeted disruption of the iNOS gene (knockout iNOS), generated as described previously (18), were generously provided by J.D. MacMicking (Cornell University Medical College, New York., USA), C. Nathan (Cornell University Medical College), and J.S. Mudgett (Merck Research Laboratories, Rahway, New Jersey, USA). iNOS-deficient mice were mated with C57BL/6×129SvEv wild-type $\left(\mathrm{NOS}^{+/+}\right)$mice to produce heterozygous $\left(\mathrm{iNOS}^{+/-}\right)$iNOSdeficient mice. $\mathrm{NOS}^{+/-}$mice were then mated with $\mathrm{iNOS}^{+/+}$mice for eight subsequent generations. $\mathrm{NOSS}^{+/-}$ mice of the ninth generation were mated with another to provide iNOS-deficient mice ( $\mathrm{NOS}^{-/-}$) and wild-type littermates $\left(\mathrm{NOS}^{+/+}\right)$with the same genetic background. The animals used in the experiment were between generations 10 and 15 bred continuously from these iNOS 
knockout and wild-type littermates. Genotyping to verify the absence or presence of the iNOS gene, or of the targeting vector, was accomplished by PCR of DNA from tail biopsies. The animals were given food and water ad libitum and maintained under pathogen-free conditions of 12-hour/12-hour light/dark.

Murine model of oxygen-induced retinopathy of prematurity. All procedures were conducted in accordance with the guidelines of the Association for Research in Vision and Ophthalmology. C57BL/6×129SvEv iNOS ${ }^{+/+}$and $i \mathrm{NOS}^{-/-}$ mice at postnatal day 7 (P7) were exposed, with their mothers, for 5 days to hyperoxic conditions (75\%), inducing vaso-obliteration and subsequent capillary loss of the central retinal vasculature. At P12, the mice were returned to room-air conditions, and extensive vitreal neovascularization occurred in $100 \%$ of NOS $^{+/+}$mice (19).

iNOS inbibition in oxygen-induced retinopathy. Oxygenincubated C57BL/6×129SvEv iNOS ${ }^{+/+}$mice were subcutaneously injected from P12 to P17 at 8-hour intervals, with $100 \mu \mathrm{l}$ of $1 \mathrm{mg} / \mathrm{ml}$ 1400W $(n=6)$ (Calbiochem, France Biochem, Meudon, France), a highly specific inhibitor of iNOS (20) in $\mathrm{H}_{2} \mathrm{O}$ or $0.9 \%$ $\mathrm{NaCl}$ solution $(n=6)$. At P17, the mice were perfused with FITC-dextran $10^{6}$ in PBS solution and their eyes enucleated. The right eyes were subjected to ex vivo angiography, and the left eyes, to intravitreal neovascularization quantification (see later here).

$R N A$ isolation and RT-PCR analysis. Total RNA from the retina was isolated at different times during and after oxygen exposure by the acid-guanidinium-thiocyanatephenol-chloroform method (21). A total of $1 \mu \mathrm{g}$ RNA was reverse transcribed for 90 minutes at $42^{\circ} \mathrm{C}$ with 200 $\mathrm{U}$ of superscript Moloney Murine Leukemia virus RT (Life Technologies SARL, Cergy-Pontoise, France), using random hexamers (21). Two microliters of cDNA was added to each PCR reaction. Amplification was performed as follows: $94^{\circ} \mathrm{C}$ for 2 minutes; 24 cycles for GAPDH, and 30 cycles for iNOS (number of cycles below the saturating conditions) at $94^{\circ} \mathrm{C}$ for 30 seconds, $55^{\circ} \mathrm{C}$ for 30 seconds, $72^{\circ} \mathrm{C}$ for 45 seconds, and then $72^{\circ} \mathrm{C}$ for 2 minutes. The amplified fragments were separated in a $1.2 \%$ agarose gel and transferred onto a nylon membrane (Amersham Pharmacia, Meudon, France). Specificity of the amplification process was verified by hybridization of blots with a ${ }^{32}$ P-labeled specific internal oligonucleotide probe. The fragments were then washed three times in $1 \times \mathrm{SSC} 0.1 \% \mathrm{SDS}$ at $50^{\circ} \mathrm{C}$. Visualization was achieved by exposure of $x$-ray film to the fragments. The nucleotide sequences of the oligonucleotide primers used for RT-PCR and those of hybridization probes are as follows: iNOS antisense exon 7 (TGTGTCTGCAGATGTGCTGAAAC); iNOS sense exon 2 (TTTCTCTTCAAAGTCAAATCCTACCA); iNOS hybridization probe (GGGTCGATGTCACATGCAGCTTGTCCAGGGA); GAPDH antisense (ATGGCATGGACTGTGGTCAT); GAPDH sense (ATGCCCCCATGTTTGTGATG); and GAPDH hybridization probe (GCTGACAATCTTGAGGGAGTTGTCATATTT).

The experiment was performed on a minimum of three independent samples of each group.
In situ RT-PCR. The eyes were enucleated at P14, immediately frozen in OCT (Tissue Tek, Puteaux, France) and sectioned $(10 \mu \mathrm{m})$. $\mathrm{NNOS}^{+/+}$and $\mathrm{iNOS}^{-/-}$sections were collected on the same silane-coated glass slides, thus undergoing the same procedure. The sections were fixed with a $10 \%$ formalin/PBS solution for 1 hour, washed three times in a $0.1 \%$ Triton X-100 PBS solution, made permeable by immersion for $10 \mathrm{~min}$ utes at $20^{\circ} \mathrm{C}$ in a $2: 1$ ethanol acetic acid solution, washed three times, and then dehydrated in alcohol. RNA was reverse transcribed for 60 minutes at $42^{\circ} \mathrm{C}$ with $200 \mathrm{U}$ of superscript Moloney Murine Leukemia virus RT (Life Technologies SARL), using random hexamers. PCR was carried out with iNOS specific primers (see RT-PCR) in the presence of Biotin16-dUTP (Boehringer Mannheim, Meylan, France) in a 1:19 ratio to dTTP under the following conditions: $94^{\circ} \mathrm{C}$ for 1 minute, $55^{\circ} \mathrm{C}$ for 1 minute, and $72^{\circ} \mathrm{C}$ for 1 minute (22). Thirty cycles were carried out. The slides were subsequently washed three times, incubated for 1 hour in a 1/100 ExtrAvidin Alkaline Phosphatase Conjugate (Sigma-Aldrich, St. Quentin Fallavier, France) PBS solution and washed again. The signal was revealed with Fast Red (Sigma-Aldrich). Amplification of the genomic iNOS sequence was avoided by choosing two oligonucleotides on exon 2 and 7, i.e., separated by five sizable introns. In the absence of Taq-enzyme, specific primers, or when PCR was carried out omitting the RT reaction, no signal was detected in oxygenated $i \mathrm{NOS}^{+/+}$ eyes. Endothelial-cell staining on adjacent sections was performed as described in the immunohistochemistry procedures. The experiment was performed on at least three independent samples of each group.

NOS activity by NADPH-diaphorase histochemistry. The eyes were enucleated at P14, immediately frozen in OCT (Tissue Tek) and sectioned $(10 \mu \mathrm{m})$. They were fixed in 4\% paraformaldehyde for 30 minutes at room temperature to reveal only NOS NADPH-diaphorase derived activity $(23,24)$. The sections were then incubated in a $0.25 \mathrm{~g} / 1$ nitroblue tetrazolium and $1 \mathrm{~g} / 1 \beta$-NADPH $0.3 \%$ Triton X-100 PBS (all from Sigma-Aldrich). The histochemical reaction was stopped by washing in PBS. Sections were then coverslipped in glycerol/PBS. The experiment was performed on a minimum of three independent samples of each group.

Ex vivo angiography. Mice were perfused at different time points with FITC-dextran $10^{6}$ in PBS solution (19). Eyes were enucleated and fixed for 30 minutes in PBS/paraformaldehyde 4\%. The retinae were dissected and flat-mounted in glycerol/PBS and viewed and photographed by fluorescence microscopy. The photographs of flat-mounted retinae were scanned. Both the total surface and the surface of the capillary-free area were measured using a computerized image-analysis system (Biocom, Les Ulis, France).

Quantification of vitreal neovascularization. The eyes were enucleated at different time points, fixed in Bouin for 24 hours, and embedded in paraffin. Serial sections (7 $\mu \mathrm{m})$ were cut sagittally parallel to the optic nerve and 
stained with PAS and Hemalun. Vascular cell nuclei found on the vitreal side of the inner limiting membrane were then counted on four sections $20 \mu \mathrm{m}$ apart from one another on each side of the optic nerve (19). Investigators carried out counting unaware of the samples' provenance. No vascular cell nucleus anterior to the internal limiting membrane was found in room air-raised animals.

Immunobistochemical analysis. P14 $\mathrm{iNOS}^{+/+}$and $\mathrm{NOS}^{-/-}$ mouse eyes were enucleated, immediately frozen in OCT and sectioned $(10 \mu \mathrm{m})$. iNOS ${ }^{-/-}$and $\mathrm{iNOS}^{+/+} \mathrm{sec}-$ tions crossing the optic nerve were collected on the same glass slides, thus undergoing the same immunohistochemical procedures. Before immunohistochemistry, they were fixed with methanol for 10 minutes at $-20^{\circ} \mathrm{C}$. The slides for eNOS and nNOS detection were incubated overnight with polyclonal anti-eNOS and nNOS (Transduction Laboratories/TEBU, Le Perray en Yvelines, France) antibody diluted at $1 / 100$ in $0.1 \%$ PBS Triton X-100 (wt/vol) at $4^{\circ} \mathrm{C}$. The slides for VEGFR2 detection were incubated for 1 hour at room temperature with rat monoclonal anti-VEGFR2 antibody (Chemicon International, Temecula, California, USA) diluted at 1/500 and biotinylated lectin griffonia simplicifolia (Sigma-Aldrich) diluted at 1/100. After washing, the sections for eNOS and nNOS detection were incubated in a solution of $1 / 100$ of secondary goat antirabbit antibody conjugated to an alkaline phosphatase (Biosys, Compiegne, France) for 60 minutes, followed by revelation with Fast-Red (Sigma-Aldrich). The sections for VEGFR2 and endothelial cell double labeling were incubated in a solution of 1/250 FITC-labeled secondary antibody (rabbit anti-rat IgG) and streptavidin TRITC for 60 minutes. The slides were than washed, mounted, and viewed and photographed with a conventional microscope for eNOS and nNOS and with a Zeiss LSM 510 LASER scanning microscope (Carl Zeiss SA, Le Pecq, France) for VEGFR2. Control experiments omitting the first antibody gave no staining (data not shown). The experiment was performed on a minimum of three independent samples of each group.

Statistical analysis. Results were expressed as mean \pm SEM. As populations were of normal distribution and equal variance, statistical analyses were performed using the Student's $t$ test.

\section{Results}

iNOS $m R N A$ detection by in vitro RT-PCR of retinal extracts. To determine whether iNOS is associated with the murine model of retinopathy of prematurity, iNOS mRNA expression in the retina was examined by in vitro RT-PCR analysis. The PCR product showed a band at the expected size of $650 \mathrm{bp}$, the specificity of which was proved by hybridization with a radioactively labeled iNOS-specific internal oligonucleotide probe (Figure 1). The iNOS signal was absent in $i \mathrm{NOS}^{+/+}$mice raised in room air. Expression of iNOS mRNA was detected in $\mathrm{iNOS}^{+/+}$oxygen-incubated retina at $\mathrm{P} 14$ and P17, corresponding to 48 and 120 hours of hypoxia, respectively. A minimal expression could also be detected at P12, before the induction of relative hypoxia. No expression was detected in $i \mathrm{NOS}^{-/-}$mice, experimental or control group at any time.

NOS isoform expression and NOS activity in P14 retina. Using a RT-PCR in situ technique, we localized the cells expressing iNOS mRNA on sections of oxygen-incubated $i \mathrm{NOS}^{+/+}$ mice in the retina at P14, when iNOS expression was strongest. The RT-PCR in situ revealed iNOS expression in the cytoplasm of cells of the inner nuclear layer (INL) (Figure 2a). Oxygen-exposed NOS $^{-/-}$and room air-raised ${ }_{i N O S}^{+/+}$mice did not show any positive cells (Figure 2, b and c, respectively). The signal on sections of oxygen-incubated $\mathrm{NOSS}^{+/+}$mice was found in only the central part of the retina (Figure $2 \mathrm{~d}$ ). Staining of endothelial cells with lectin griffonia simplicifolia on adjacent sections (Figure 2e) revealed that this area corresponds precisely to the central ischemic area generated by the oxygen exposure.

eNOS was detected by immunohistochemistry at P14 in endothelial cells of room air-raised $\mathrm{NOS}^{+/+}$mice (Figure 2k) throughout the retina, which at this stage is entirely vascularized. In oxygen-incubated $i \mathrm{NOS}^{+/+}$ and $\mathrm{iNOS}^{-/-}$mice at P14, eNOS was expressed in endothelial cells of the vascularized periphery (data not shown), whereas no expression of eNOS could be detected in the capillary-free center of oxygen-incubated $\mathrm{NNOS}^{+/+}$(Figure 2i) and $i \mathrm{NOS}^{-/-}$mice (Figure $2 \mathrm{j}$ ).

nNOS expression was detected by immunohistochemistry at P14 in the ganglion cell layer and nerve fiber layer of oxygen-incubated $\mathrm{NOOS}^{+/+}$and $i \mathrm{NOS}^{-/-}$ mice as well as in room air-raised $\mathrm{NOSS}^{+/+}$mice (Figure $2,1, \mathrm{~m}$, and $\mathrm{n}$, respectively) throughout the retina. The expression in room air-raised mice seemed to be slightly weaker than in oxygen-exposed $i \mathrm{NOS}^{+/+}$and $i \mathrm{NOS}^{-/-}$ animals. No differences could be detected in the expression pattern or in signal intensity between oxygen-incubated $i \mathrm{NOS}^{+/+}$and $\mathrm{iNOS}^{-/-}$mice.

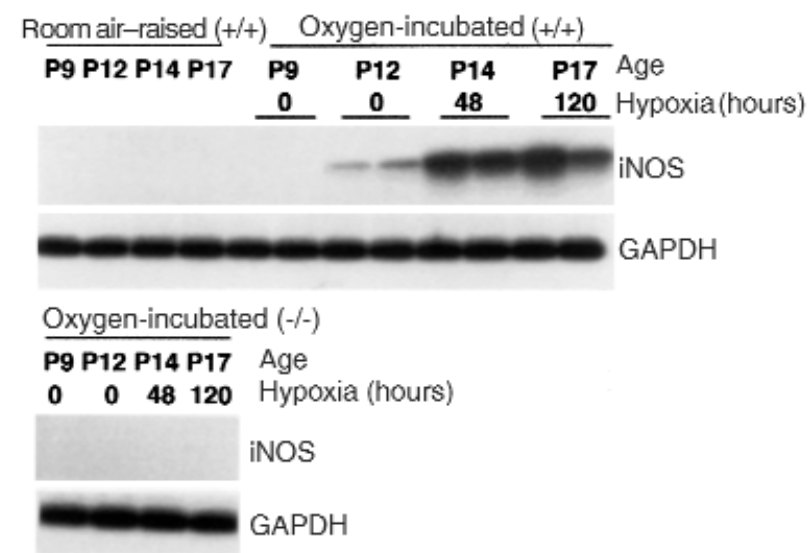

Figure 1

RT-PCR analysis of iNOS mRNA expression in room air-raised NOS $^{+/+}$ and oxygen-incubated $\mathrm{NOS}^{+/+}$and $\mathrm{NNOS}^{-/-}$mice. The iNOS signal is absent in $\mathrm{NOOS}^{+/+}$mice raised in room air. iNOS mRNA is expressed in oxygen-incubated $\mathrm{NOSS}^{+/+}$retinae at postnatal day (P) 14 and P17. No expression was detected in oxygen-incubated iNOS mice. 
To correlate NOS expression with NOS activity we performed NADPH-diaphorase histochemistry on PAF 4\% fixed sections at P14, revealing NOS NADPHdiaphorase activity (24). A signal in the ganglion layer and nerve fiber layer was observed on all sections, being slightly weaker in room air-raised $\mathrm{NOS}^{+/+}$mice (Figure 2h) compared with oxygen-incubated $i \mathrm{NOS}^{+/+}$ and $\mathrm{iNOS}^{-/-}$mice (Figure 2, f and g, respectively). This distribution corresponds to the nNOS immunohistochemical signal (Figure 2, 1-n). Room air-raised $i \mathrm{NOS}^{+/+}$mice also exhibited a signal in a vessel-like distribution throughout the retina (Figure $2 \mathrm{~h}$, showing the central aspect), paralleling the eNOS immunohistochemical signal in room air-raised $\mathrm{NOSS}^{+/+}$mice (Figure $2 \mathrm{k}$ ). This signal pattern was only detected in the vascularized periphery of oxygen-incubated $\mathrm{iNOS}^{+/+}$ and $\mathrm{NNOS}^{-/-}$mice (data not shown), but not in the capillary-free central area (Figure 2, f and g). Furthermore, we observed a signal in the central, ischemic retina of oxygen-incubated $\mathrm{NOS}^{+/+}$mice in the INL (Figure 2f, arrowheads) that could not be found in either oxygenincubated $i \mathrm{NOS}^{-/-}$(Figure 2g) or room air-raised $i \mathrm{NOS}^{+/+}$mice (Figure $2 \mathrm{~h}$ ). This pattern corresponds precisely to the expression pattern of iNOS mRNA in oxygen-incubated $\mathrm{NOOS}^{+/+}$mice (Figure 2a), indicating iNOS specific activity.

Increased intraretinal angiogenesis in iNOS ${ }^{-/-}$mice. To investigate the influence of iNOS on intraretinal vascularization we compared the capillary-free area as a percentage of total retinal surface in FITCdextran-perfused retina of oxygen-incubated $\mathrm{NOOS}^{+/+}$ and $i \mathrm{NOS}^{-/-}$mice at different stages. On P12, after 5 days of oxygen exposure, no difference in the size of the capillary-free area in either $i \mathrm{NOS}^{+/+}$or $i \mathrm{NOS}^{-/-}$mice was detectable (Figure 3, a and d, respectively). No significant difference could be observed at P14 after 48 hours of hypoxia (Figure $3, \mathrm{~b}\left[\mathrm{iNOS}^{+/+}\right]$and e $\left[\mathrm{iNOS}^{-/}\right]$).

However, retinal flat-mounts at P17 (120-hour hypoxia) showed a significant reduction in the size of the central capillary-free area and a relatively normal vascular morphology in $\mathrm{NOS}^{-/-}$mice (Figure 3f). On the other hand, retina of $i \mathrm{NOS}^{+/+}$mice at P17 had persisting avascular areas $(P<0.0001 ; n=6)$ bordered by active intravitreal neovascularization (Figure $3 c$ ).

Reduction of vitreal neovascularization in iNOS ${ }^{-/-}$mice. Intravitreal neovascularization was assessed on P14, $\mathrm{P} 17$, and P20 by counting intravitreal vascular nuclei on serial sections (Figure 3j). Room air-raised NOS $^{+/+}$ and $i \mathrm{NOS}^{-/-}$mice showed less than one intravitreal nucleus per section. On P14, a weak equivalent neovascular response was detected in both $\mathrm{NOS}^{+/+}$and iNOS $^{-1-}$ oxygen-exposed mice $(n=5)$. At P17, the intravitreal neovascularization was significantly stronger in the $\mathrm{NOSS}^{+/+}$mice (Figure $3 \mathrm{~h}$ ) compared with the $\mathrm{NNOS}^{-/-}$mice (Figure $\left.3 \mathrm{i}\right)(P<0.0001 ; n=10)$. Counts at P20 also revealed a highly significant difference $(P<0.0001 ; n=5)$. Both types of mouse reached a maximal neovascularization at P17 as described for the $i \mathrm{NOS}^{+/+}$group (19).
VEGFR2 expression in $\mathrm{iNOS}^{+/+}$and $\mathrm{NNOS}^{-/-}$mice. It has been demonstrated that VEGF (25) and the VEGF receptor type 2 (flk1/kdr/VEGFR2) (26) are very important mediators of angiogenesis in ischemic conditions. To assess the influence of iNOS-related NO production on VEGFR2 expression, we performed immunohistochemistry of VEGFR2-endothelial cell double labeling on eye sections including the optic nerve of room air-raised and oxygen-incubated $\mathrm{NOS}^{+/+}$and $\mathrm{iNOS}^{-/-}$ mice. We analyzed eyes at P14, before any significant differences in the vascular pattern of the experimental animals had occurred.

Room air-raised mice expressed VEGFR2 (Figure 4, g and $h$ ) in vascular endothelial cells as demonstrated by double staining (Figure 4, g and i), and more weakly in the nerve fiber layer. Furthermore, the staining pattern of VEGFR2 throughout the retina (Figure 4h) in a Müller cell-like distribution suggests that these cells express VEGFR2 as well. The vascularized periphery of oxygen-incubated $i \mathrm{NOS}^{+/+}$mice revealed a similar staining pattern to that of room air-raised mice (Figure 4a, left). However, in the central ischemic retina, VEGFR2 expression in the nerve fiber layer was downregulated in oxygen-incubated $\mathrm{NOS}^{+/+}$mice (Figure 4a, between arrowheads). Furthermore, VEGFR2 expression in endothelial cells adjacent to the ischemic area was decreased (Figure 4, b and c, double labeling) in oxygenincubated $\mathrm{NOS}^{+/+}$mice. On the other hand, VEGFR2 expression in oxygen-incubated $\mathrm{NOS}^{-/-}$mice was upregulated in the nerve fiber layer of the ischemic retina (Figure $4 \mathrm{~d}$, between arrowheads) and in endothelial cells of vessels on the edge of the avascular area (Figure 4, e and $f$, double labeling) compared with room air-raised mice. The difference compared with oxygen-incubated $\mathrm{NOS}^{+/+}$ animals was even greater. VEGFR2 expression in the retinal periphery was similar in $\mathrm{NOS}^{+/+}$and $\mathrm{NNOS}^{-/-}$mice. Double labeling with glial fibrillary acidic protein (GFAP) identified the VEGFR2-expressing cells in the nerve fiber layer as astrocytes (data not shown).

$i N O S$ inbibition in oxygen-incubated $\mathrm{NOS}^{+/+}$mice increases intraretinal angiogenesis and reduces vitreal neovascularization. To evaluate the potential utility of iNOS inhibitors for therapeutic use in ischemic retinopathy, we tested the effect of the highly potent iNOS inhibitor 1400W (20) on our model. Littermates of oxygen-incubated $\mathrm{NOS}^{+/+}$mice were injected subcutaneously every 8 hours with either $1400 \mathrm{~W}$ ( $100 \mu \mathrm{l}$ of a $1 \mathrm{mg} / \mathrm{ml}$ solution) or $\mathrm{NaCl}$ from P12 to P17, to coincide with detectable iNOS expression. Retinal flat-mounts at P17 (120-hour hypoxia) showed a significant reduction in the size of the central capillary-free area in $1400 \mathrm{~W}$-treated $\mathrm{NOS}^{+/+}$mice compared with the $\mathrm{NaCl}$-injected control group $(P=0.0006 ; n=6)$ (Figure $5 a)$. Furthermore, the intravitreal neovascularization at P17 was significantly reduced in $1400 \mathrm{~W}$-treated mice compared with control $(P<0.0001 ; n=6)$ (Figure 5b).

\section{Discussion}

These data demonstrate that iNOS is induced in ischemic proliferative retinopathy. RT-PCR assays 


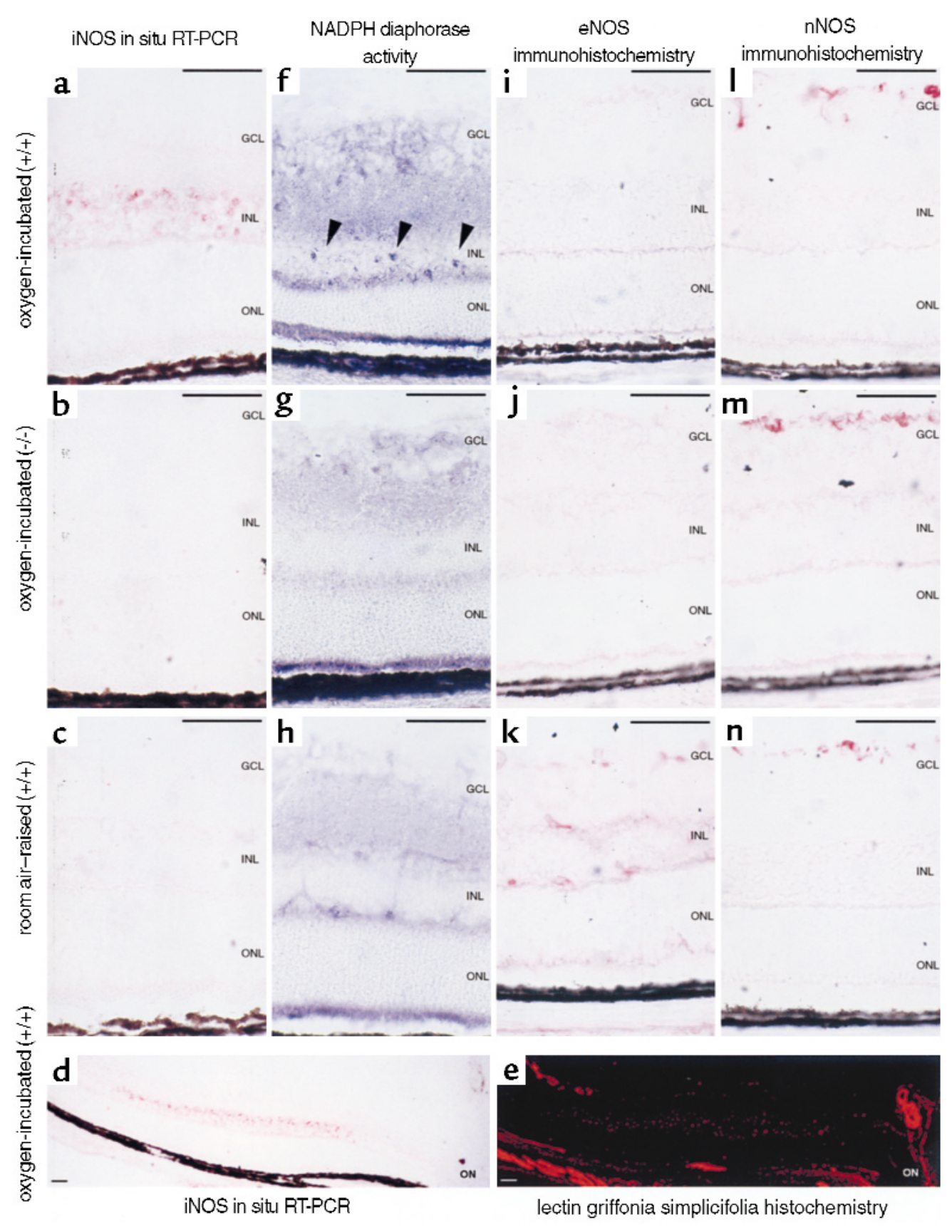

\section{Figure 2}

NOS isoform expression and NOS activity in the central P14 retina. iNOS is expressed in the inner nuclear layer (INL) of oxygen-incubated $\mathrm{iNOS}^{+/+}$sections (a). Oxygen-exposed $\mathrm{NNOS}^{-/-}$(b) and room air-raised $i \mathrm{NOS}^{+/+}$mice (c) do not show any positive cells. The signal on sections of oxygen-incubated $i \mathrm{NOS}^{+/+}$mice is restricted to the INL of the central retina (d). Endothelial-cell staining on adjacent sections (e) reveals that this area corresponds precisely to the central ischemic area. NOS activity is detected by NADPH-diaphorase activity in the ganglion layer and nerve fiber layer on all sections, being slightly stronger in oxygen-incubated iNOS i $^{/+}$and $i N O S^{-/-}$mice (f and $\mathbf{g}$, respectively) compared with room air-raised iNOS ${ }^{+/+}$mice $(\mathbf{h})$, probably corresponding to nNOS expression (I-n). Room air-raised $\mathrm{iNOS}^{+/+}$mice also exhibit a signal in a vessel-like distribution (h), probably corresponding to eNOS expression (k). Furthermore, we observe a signal in the central, ischemic retina of oxygen-incubated iNOS ${ }^{++}$mice in the INL (f, arrowheads), This pattern corresponds to the expression pattern of iNOS in oxygen-incubated NOS $^{+/+}$mice (a); neither eNOS (i) nor nNOS (I) expression is found in this distribution in oxygen-incubated $\mathrm{NOS}^{+/+}$mice, suggesting iNOS-specific activity. GCL, ganglion cell layer; INL, inner nuclear layer; ONL, outer nuclear layer; Ch, choroide; ON, optic nerve. Scale bar $=50 \mu \mathrm{m}$. 
showed that iNOS mRNA is mainly expressed at P14 and P17 in the ischemic phase. iNOS induction by hypoxia has been demonstrated by others in vitro (27) and in vivo (28). Hypoxia inducible factor (HIF) and nuclear factor kappa B (NF- $\mathrm{KB})$ are upregulated in the murine model of ischemic proliferative retinopathy $(29,30)$. Because consensus sequences for these two transcription factors are present in the promoter of iNOS $(7,27)$, HIF and NF- $\kappa$ B could be responsible for iNOS induction in the ischemic retina.

With regard to the origin of iNOS in the retina in this model, RT-PCR in situ experiments revealed the presence of iNOS mRNA in the cytoplasm of cells of the INL of the central retina. This corresponds precisely to the ischemic retina, as shown by endothelial-cell labeling on adjacent sections, demonstrating that iNOS is expressed only in the ischemic retina. In addition, NOS-related NADPH-diaphorase activity occurred at P14 in the INL of the central ischemic retina of oxygeninduced $\mathrm{NNOS}^{+/+}$mice in the same pattern as iNOS mRNA. Furthermore, no eNOS or nNOS expression could be detected in the INL of the central ischemic retina of oxygen-induced $i \mathrm{NOS}^{+/+}$mice, indicating that the NADPH-diaphorase activity in these cells is not due to either eNOS or nNOS. No NADPH-diaphorase activity was detectable in the INL of the central ischemic retina of oxygen-induced $\mathrm{NOS}^{-/-}$mice either.
These results taken together suggest that the NADPHdiaphorase activity in the INL of the central ischemic retina of oxygen-induced $i \mathrm{NOS}^{+/+}$mice is due to functional iNOS protein in this model. Although double labeling could not identify the cells expressing iNOS, we detected an activation of Müller cells in the ischemic area by GFAP immunohistochemistry (data not shown). We have previously demonstrated that Müller cells express iNOS in vitro (31), and they may therefore be a major source of iNOS expression in this model. Nevertheless, other types, such as amacrine, horizontal, bipolar and microglial cells, contribute to the NO production during ischemic proliferative retinopathy.

Using mice lacking the iNOS gene, we evaluated the influence of the iNOS enzyme on neovascularization in ischemic proliferative retinopathy. iNOS had no effect on capillary loss during oxygen incubation as demonstrated by the equivalent size of the central capillary-free area at P12 in $\mathrm{NNOS}^{+/+}$and $i \mathrm{NOS}^{-/-}$mice. In contrast, after 120 hours of ischemia at P17, intraretinal neovascularization in $i \mathrm{NOS}^{-/-}$mice was significantly stronger than in $i \mathrm{NOS}^{+/+}$mice. We suggest that $\mathrm{NO}$ production due to iNOS expression in the ischemic central area significantly slows down intraretinal angiogenesis. Pathological intravitreal neovascularization was significantly reduced in $i \mathrm{NOS}^{-/-}$mice at P17 and P20 compared with $i \mathrm{NOS}^{+/+}$mice. The maximal
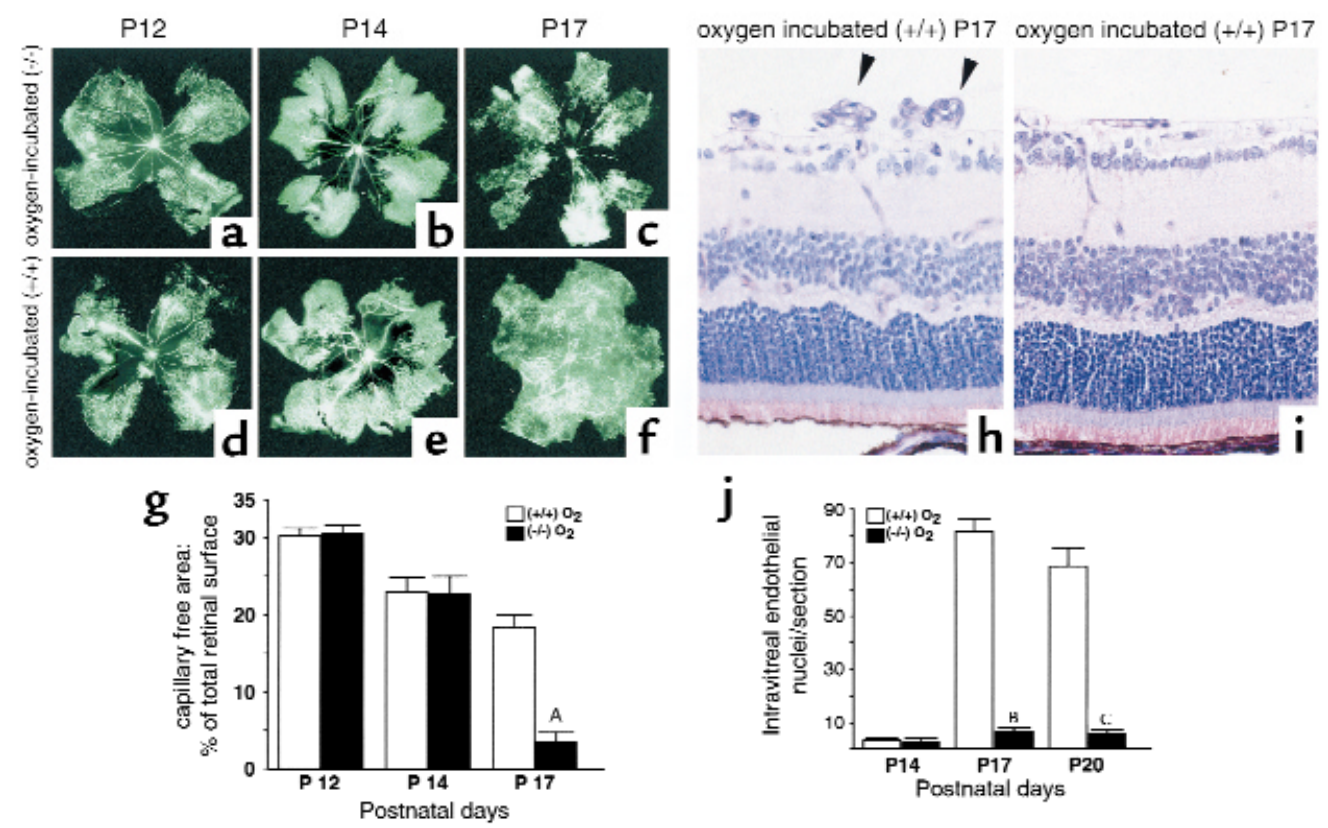

Figure 3

Quantification of ischemic retina $(\mathbf{a}-\mathbf{g})$ and intravitreal neovascularization $(\mathbf{h}-\mathbf{j})$. ( $\mathbf{g})$ Capillary-free area as a percentage of total retinal surface in FITC-dextran-perfused retinae of oxygen-incubated iNOS ${ }^{+/+}$mice $(\mathbf{a}-\mathbf{c})$ and iNOS $^{-/-}(\mathbf{d}-\mathbf{f})$ mice at different stages. On P12, no difference in the size of the capillary-free area in $\operatorname{iNOS}^{+/+}(\mathbf{a})$ and $\mathrm{NNOS}^{-/-}(\mathbf{d})$ mice is detectable. Nor is there any significant difference at P14 after 48 hours of hypoxia $\left(\mathbf{b}\left[\mathrm{iNOS}^{+/+}\right]\right.$, e $\left.\left[\mathrm{iNOS}^{-/-}\right]\right)$. At P17, retinal flat-mounts of oxygen-incubated iNOS ${ }^{-/-}$mice $(\mathbf{f})$ reveal a significant reduction in the size of the central capillary-free area and a relatively normal vascular morphology compared with retinae of oxygen-incubated ${ }_{i N O S}{ }^{+/+}$mice $(\mathbf{c}) .{ }^{A} P<0.0001 ; n=6$. Picture side length $\mathbf{a}-\mathbf{f}=5.150 \mathrm{~mm}$. (j) Intravitreal neovascularization quantified by endothelial cell nucleus count on serial sections. On P14, a weak equivalent neovascular response is detected in $\mathrm{iNOS}^{+/+}$and iNOS $^{-/-}$oxygen-exposed mice $(n=5)$. At P17, the intravitreal neovascularization is significantly stronger in the $i N O S^{+/+}$mice (h, arrowheads) compared with iNOS ${ }^{-/-}$mice (i). ${ }^{\mathrm{B}} P<0.0001 ; n=10$. Counts at $\mathrm{P} 20$ reveal a similar difference $\left({ }^{C} P<0.0001 ; n=5\right)$. Picture side width $(\mathbf{h}, \mathbf{i})=220 \mu \mathrm{m}$. 

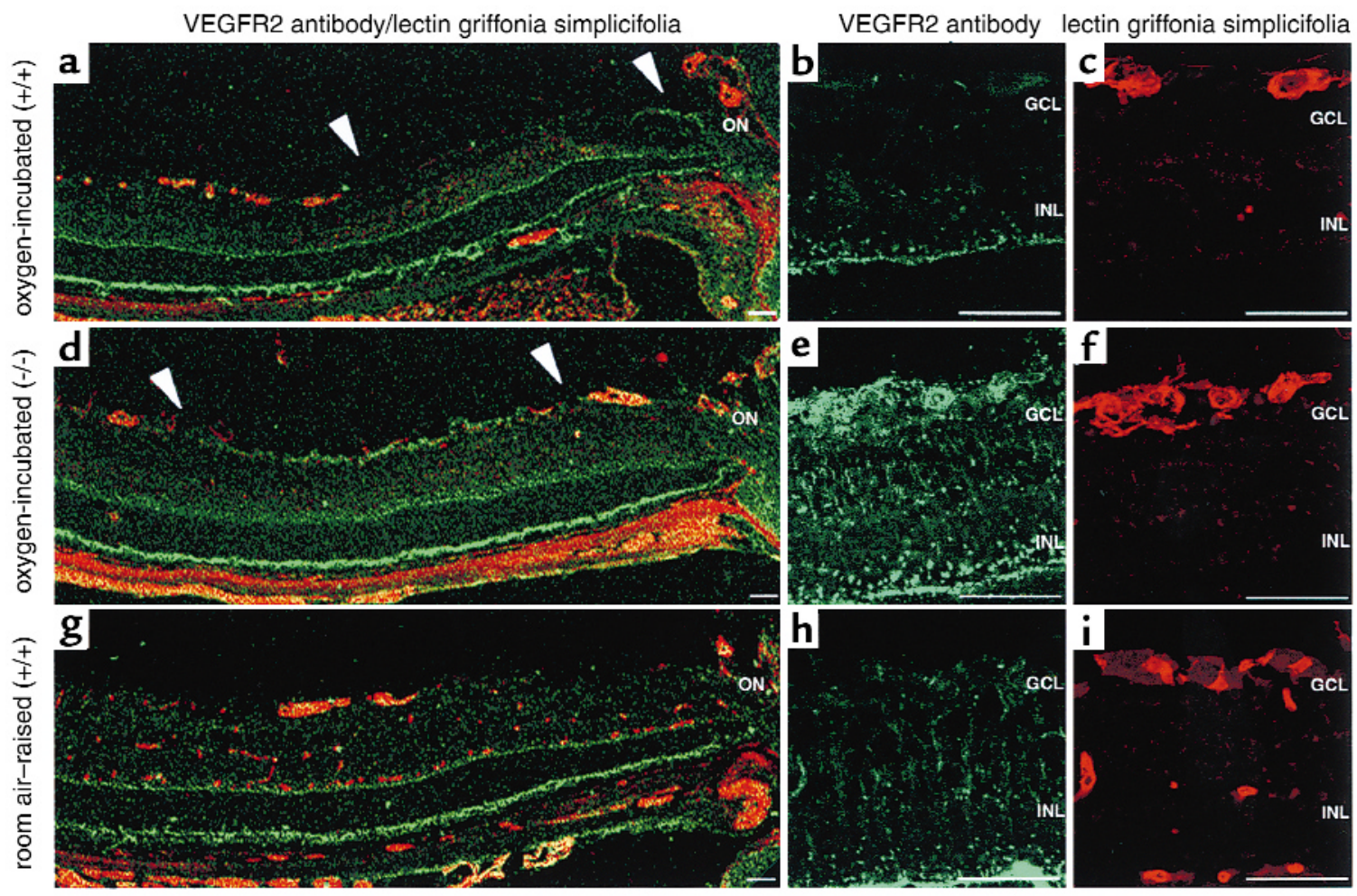

Figure 4

VEGFR2, lectin griffonia simplicifolia (endothelial cell) immunohistochemistry on central P14 retina. (a-c) Oxygen-incubated iNOS $/++$ eyes; (d-f) oxygen-incubated $\mathrm{NNOS}^{-/-}$eyes; (g-h) room air-raised $\mathrm{NOS}^{+/+}$mice. VEGFR2 (green) and lectin griffonia simplicifolia (red) double labeling immunohistochemistry is shown. Room air-raised mice express VEGFR2 in vascular endothelial cells, weakly in astrocytes and Müller cells $\left(\mathbf{g}\right.$ and $\mathbf{h}$ ). In oxygen-incubated $\mathrm{NOS}^{+/+}$mice, VEGFR2 expression is downregulated in astrocytes of the central ischemic retina (a, between arrowheads) and in endothelial cells adjacent to the ischemic area (b and c, double labeling). In contrast, VEGFR2 expression in oxygenated NOS $^{-/-}$eyes is upregulated in astrocytes of the ischemic area ( $\mathbf{d}$, between arrowheads) and in endothelial cells of vessels on the edge of the avascular area (e and f, double labeling). GCL, ganglion cell layer; INL, inner nuclear layer; ON, optic nerve. Scale bar $=50 \mu \mathrm{m}$.

intravitreal neovascular response in oxygen-incubated $i \mathrm{NOS}^{-/-}$was reached at $\mathrm{P} 17$, as seen in the $i \mathrm{NOS}^{+/+}$mice. This shows that the difference in intravitreal neovascularization in $\mathrm{NOS}^{+/+}$and $i \mathrm{NOS}^{-/-}$mice is not simply due to a retardation in intravitreal neovascularization in the $i \mathrm{NOS}^{-/-}$mice but remains over time. The difference in pathological intravitreal neovascularization in $\mathrm{iNOS}^{+/+}$and $\mathrm{NNOS}^{-/-}$animals is surprising, as intravitreal neovascularization develops up to $1,500 \mu \mathrm{m}$ away from the site of iNOS-related NO production in $i \mathrm{NOS}^{+/+}$mice. In view of long diffusion distances, intravitreal neovascularization in iNOS-expressing mice should be exposed to considerably lower NO concentrations compared with intraretinal neovascularization, which occurs closer to the ischemic area where iNOS is expressed. We therefore postulate that the primary effect of iNOS is the inhibition of vascularization in the ischemic retina; we hypothesize that the promotion of intravitreal neovascularization occurs secondarily owing to the persistence of the ischemic area.

To understand the mechanisms by which iNOS expression inhibits intraretinal neovascularization and encourages intravitreal neovascularization, we analyzed VEGFR2 expression in $i \mathrm{NOS}^{+/+}$and $\mathrm{NOS}^{-/-}$mice by immunohistochemistry. VEGF has been shown to be a very important mediator of physiological and pathological angiogenesis in the retina $(2,32,33)$ and more specifically in the murine model of ischemic proliferative retinopathy (25). Its proliferative and migratory effects have been reported to be mediated by VEGFR2 (26). NO has been shown to downregulate VEGF and VEGFR2 in vitro $(12,34,35)$ and in vivo $(1,13)$ in other models.

We chose to analyze sections at P14 because at this relatively early stage in the ischemic phase no significant differences in the size of the capillary-free area or in the intravitreal neovascular response could be detected; thus differences in VEGFR2 expression could not be ascribed to differences of the ischemic state in $i \mathrm{NOS}^{+/+}$ and $i \mathrm{NOS}^{-/-}$mice. VEGFR2 was expressed in room air-raised P14 mice in vascular endothelial cells, the ganglion cell layer, the INL, and the outer nuclear layer, as described previously (36). We identified the nonvascular cells expressing VEGFR2 in the nerve fiber layer to be astrocytes, by double labeling with GFAP antibody. Astrocytes play a crucial role in the organization of physiological retinal vasculogenesis and angiogenesis. It has been suggested that their dysfunction is of importance in ischemic retinal neovascularization (37).

In comparison with $i \mathrm{NOS}^{-/-}$mice, in the ischemic retina of $i \mathrm{NOS}^{+/+}$mice VEGFR2 expression adjacent to 
a

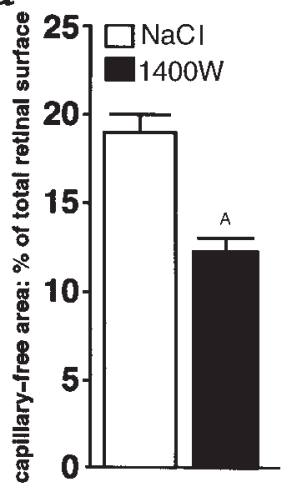

b

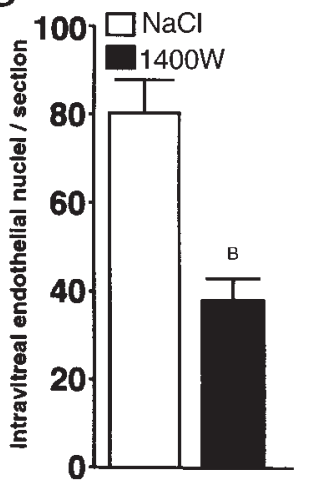

Figure 5

iNOS inhibition in oxygen-incubated $\mathrm{NOS}^{+/+}$mice. $\mathrm{iNOS}^{+/+}$littermates were treated with the iNOS inhibitor $1400 \mathrm{~W}$ or $\mathrm{NaCl}$. Retinal flatmounts at P17 show a significant reduction in the size of the central capillary-free area in $1400 \mathrm{~W}$-treated $\mathrm{NOS}^{+/+}$mice compared with the $\mathrm{NaCl}$-injected control group $\left({ }^{A} P=0.0006 ; n=6\right)(\mathbf{a})$. Furthermore, the intravitreal neovascularization at $\mathrm{P} 17$ is significantly reduced in $1400 \mathrm{~W}$-treated mice compared with control $\left({ }^{\mathrm{B}} P<0.0001 ; n=6\right)(\mathbf{b})$.

iNOS-expressing cells was much weaker, notably in endothelial cells of vessels on the edge of the avascular area and in astrocytes in the central avascular retina. On the other hand, VEGFR2 expression in endothelial cells and astrocytes in the peripheral retina remained unaffected. There was no significant loss of glial cells (GFAP) in the ischemic area of $\mathrm{NNOS}^{+/+}$mice (data not shown), nor any difference in the vascular staining pattern (lectin griffonia simplicifolia), suggesting a downregulation of VEGFR2 in these cells rather than a diminished signal due to cell depletion.

iNOS localization in the ischemic retina leads us to postulate that the primary effect of iNOS expression is the inhibition of angiogenesis in the ischemic tissue. We suggest that neovascularization from the vascularized retina into the iNOS-expressing ischemic retina of $i \mathrm{NOS}^{+/+}$mice is inhibited, at least in part, because VEGFR2 is downregulated in vascular endothelial cells directly adjacent to iNOS-expressing cells in the ischemic retina. These endothelial cells, from which the revascularization of the ischemic retina occurs, would in consequence become less responsive to the VEGF stimulus. Furthermore, the downregulation of VEGFR2 in astrocytes throughout the ischemic retina points toward a dysfunction of this type of cell in the ischemic retina of iNOS-expressing mice, which could further slow down the revascularization of the ischemic retina in the $\mathrm{NNOS}^{+/+}$mice.

The intravitreal neovascularizations occurring in the midperipheral and peripheral retina are relatively far away from the iNOS expression site (up to $1,500 \mu \mathrm{m}$ ) and therefore exposed to a considerably lower NO concentration compared with the ischemic retina, where iNOS is expressed. It would appear that endothelial cells with uninhibited VEGFR2 expression (as observed in our experiments) are more responsive to the VEGF

stimulus and therefore proliferate in the peripheral retina and locally invade the vitreous under the hypoxic stimulus. From the inhibition of the revascularization of the ischemic retina, we infer that angiogenic factors stay upregulated and encourage peripheral intravitreal neovascularization further. We therefore suggest that the differences observed in intravitreal neovascularization occur secondarily to the inhibition of vascularization in the ischemic retina.

Regarding the potential role of VEGF in this model, we also analyzed VEGF expression at P14 in oxygen incubated $\mathrm{iNOS}^{+/+}$and $i \mathrm{NOS}^{-/-}$mice. VEGF expression in ganglion cells of the ischemic retina of $i \mathrm{NOS}^{+/+}$mice also seemed to be weaker compared with $i \mathrm{NOS}^{-/-}$mice, whereas VEGF expression in the periphery of the two types of mice was unaffected (F. Sennlaub and O. Goureau, unpublished data). Although the differences observed in VEGF expression were less marked than those found in VEGFR2 expression, they may well contribute to the NO-mediated inhibition of the vascularization in the hypoxic area in ischemic retinopathy.

An overall antiangiogenic therapy in ischemic retinopathy, blocking intraretinal as well as intravitreal angiogenesis, inhibits pathological intravitreal neovascularization, but will also keep the retina ischemic. Ideally, a therapy for ischemic retinopathy should encourage angiogenesis in the ischemic retina, improve the hypoxic state of the tissue, and inhibit pathological intravitreal neovascularization, the situation found in our iNOS-deficient animals. To test the feasibility of therapy, we therefore treated oxygen-incubated $\mathrm{NOS}^{+/+}$mice with the potent iNOS inhibitor 1400W in the ischemic phase (P12-P17), when iNOS, according to our results, is expressed. Angiogenesis in the ischemic retina in oxygen-incubated $\mathrm{iNOS}^{+/+}$mice was found to be significantly enhanced, and pathological intravitreal neovascularization inhibited, in 1400W-treated animals compared with control mice. However, these differences observed in the $1400 \mathrm{~W}$-treated group were less significant than in the $i \mathrm{NOS}^{-/-}$animals. Although intraretinal 1400W concentrations were not determined, the local concentration of $1400 \mathrm{~W}$ in the iNOS-expressing avascular retina after subcutaneous drug administration may be too low to ensure complete iNOS inhibition. The residual iNOS activity may therefore account for the observed differences in neovascularization between the $1400 \mathrm{~W}$-treated group and $\mathrm{NOS}^{-/-}$animals.

To ensure higher $1400 \mathrm{~W}$ concentrations in the iNOSexpressing ischemic area, it would be useful to develop an efficient mode for local drug administration. Given that intravitreal administration of the molecule was unreliable in this model because of the necessity of repeated injections and anesthesia in newborn mice, we are currently investigating the possibility of topical 1400W administration in our laboratory.

In conclusion, to our knowledge, this is the first evidence that angiogenesis in the ischemic retina can be 
pharmacologically enhanced. These findings confirm that iNOS plays a crucial role in retinal neovascular diseases and further show that it is an ideal target for the control of vitreal neovascularization by improving the vascularization of the hypoxic retina. In other ischemic conditions such as cerebral ischemia, stroke, and ischemic limb disease, the inhibition of neovascularization in ischemic tissue still poses a crucial problem. The role of iNOS in angiogenesis of these diseases remains to be elucidated.

\section{Acknowledgments}

We thank Christophe Klein (Service commun d'imagerie cellulaire \& cytometrie INSERM IFR58), Sylvie Thomasseau, and Laurent Jonet for technical assistance; Hervé Coët for photographic work; Jean Claude Jeanny and Thérèse de Bizemont for helpful suggestions; and Andrew Ayers and Roni Amelan for critical review of the manuscript. This work was supported by INSERM and by grants from the Deutscher Akademischer Austauschdienst, the Fondation Paulette Darty, and le Centre de Recherche Ophtalmologique.

1. Folkman, J. 1995. Angiogenesis in cancer, vascular, rheumatoid and other disease. Nat. Med. 1:27-31.

2. Adamis, A.P., Aiello, L.P., and D'Amato, R.A. 1999. Angiogenesis and ophthalmic disease. Angiogenesis. 3:9-14.

3. Knowles, R.G., and Moncada, S. 1994. Nitric oxide synthases in mammals. Biochem. J. 298:249-258.

4. Christopherson, K.S., and Bredt, D.S. 1997. Nitric oxide in excitable tissues: physiological roles and disease. J. Clin. Invest. 100:2424-2429.

5. MacMicking, J., Xie, Q.W., and Nathan, C. 1997. Nitric oxide and macrophage function. Annu. Rev. Immunol. 15:323-350.

6. Fang, F.C. 1997. Perspectives series: host/pathogen interactions. Mechanisms of nitric oxide-related antimicrobial activity. J. Clin. Invest. 99:2818-2825.

7. Nathan, C. 1997. Inducible nitric oxide synthase: what difference does it make? J. Clin. Invest. 100:2417-2423.

8. Parenti, A., et al. 1998. Nitric oxide is an upstream signal of vascular endothelial growth factor-induced extracellular signal-regulated kinase $1 / 2$ activation in postcapillary endothelium. J. Biol. Chem. 273:4220-4226

9. Ziche, M., et al. 1997. Nitric oxide synthase lies downstream from vascular endothelial growth factor-induced but not basic fibroblast growth factor-induced angiogenesis. J. Clin. Invest. 99:2625-2634.

10. Papapetropoulos, A., Garcia-Cardena, G., Madri, J.A., and Sessa, W.C 1997. Nitric oxide production contributes to the angiogenic properties of vascular endothelial growth factor in human endothelial cells. J. Clin. Invest. 100:3131-3139.

11. Tsurumi, Y., et al. 1997. Reciprocal relation between VEGF and NO in the regulation of endothelial integrity. Nat. Med. 3:879-886.

12. Liu, Y., et al. 1998. Carbon monoxide and nitric oxide suppress the hypoxic induction of vascular endothelial growth factor gene via the 5 enhancer. J. Biol. Chem. 273:15257-15262.

13. Tuder, R.M., Flook, B.E., and Voelkel, N.F. 1995. Increased gene expression for VEGF and the VEGF receptors KDR/Flk and Flt in lungs exposed to acute or to chronic hypoxia. Modulation of gene expression by nitric oxide. J. Clin. Invest. 95:1798-1807.

14. Fulton, D., et al. 1999. Regulation of endothelium-derived nitric oxide production by the protein kinase Akt [erratum 1999, 400:792]. Nature. 399:597-601.
15. Murohara, T., et al. 1998. Nitric oxide synthase modulates angiogenesis in response to tissue ischemia. J. Clin. Invest. 101:2567-2578.

16. Sennlaub, F., Courtois, Y., and Goureau, O. 1999. Nitric oxide synthaseII is expressed in severe corneal alkali burns and inhibits neovascularization. Invest. Ophthalmol. Vis. Sci. 40:2773-2779.

17. Pipili-Synetos, E., et al. 2000. Nitric oxide synthase expression, enzyme activity and $\mathrm{NO}$ production during angiogenesis in the chick chorioallantoic membrane. Br. J. Pharmacol. 129:207-213.

18. MacMicking, J.D., et al. 1995. Altered responses to bacterial infection and endotoxic shock in mice lacking inducible nitric oxide synthase erratum 1995, 81:1170]. Cell. 81:641-650.

19. Smith, L.E., et al. 1994. Oxygen-induced retinopathy in the mouse. Invest. Ophthalmol. Vis. Sci. 35:101-111.

20. Garvey, E.P., et al. 1997. 1400W is a slow, tight binding, and highly selective inhibitor of inducible nitric-oxide synthase in vitro and in vivo. $J$. Biol. Chem. 272:4959-4963.

21. Goureau, O., Bellot, J., Thillaye, B., Courtois, Y., and de Kozak, Y. 1995. Increased nitric oxide production in endotoxin-induced uveitis. Reduction of uveitis by an inhibitor of nitric oxide synthase. J. Immunol. 154:6518-6523.

22. Thaker, V. 1999. In situ RT-PCR and hybridization techniques. Methods Mol. Biol. 115:379-402.

23. Hope, B.T., Michael, G.J., Knigge, K.M., and Vincent, S.R. 1991. Neuronal NADPH diaphorase is a nitric oxide synthase. Proc. Natl. Acad. Sci. USA. 88:2811-2814.

24. Matsumoto, T., Nakane, M., Pollock, J.S., Kuk, J.E., and Forstermann, U. 1993. A correlation between soluble brain nitric oxide synthase and NADPH-diaphorase activity is only seen after exposure of the tissue to fixative. Neurosci. Lett. 155:61-64.

25. Aiello, L.P., et al. 1995. Suppression of retinal neovascularization in vivo by inhibition of vascular endothelial growth factor (VEGF) using soluble VEGF-receptor chimeric proteins. Proc. Natl. Acad. Sci. USA. 92:10457-10461.

26. Bernatchez, P.N., Soker, S., and Sirois, M.G. 1999. Vascular endothelial growth factor effect on endothelial cell proliferation, migration, and platelet-activating factor synthesis is Flk-1-dependent. J. Biol. Chem. 274:31047-31054.

27. Melillo, G., et al. 1995. A hypoxia-responsive element mediates a novel pathway of activation of the inducible nitric oxide synthase promoter. $J$. Exp. Med. 182:1683-1693.

28. Palmer, L.A., Semenza, G.L., Stoler, M.H., and Johns, R.A. 1998. Hypoxia induces type II NOS gene expression in pulmonary artery endothelial cells via HIF-1. Am. J. Physiol. 274:L212-L219.

29. Ozaki, H., et al. 1999. Hypoxia inducible factor-1alpha is increased in ischemic retina: temporal and spatial correlation with VEGF expression. Invest. Ophthalmol. Vis. Sci. 40:182-189.

30. Yoshida, A., Yoshida, S., Ishibashi, T., Kuwano, M., and Inomata, H. 1999. Suppression of retinal neovascularization by the NF-kappaB inhibitor pyrrolidine dithiocarbamate in mice. Invest. Ophthalmol. Vis. Sci. 40:1624-1629.

31. Goureau, O., Hicks, D., Courtois, Y., and De Kozak, Y. 1994. Induction and regulation of nitric oxide synthase in retinal Muller glial cells. J. Neurochem. 63:310-317.

32. D'Amore, P.A. 1994. Mechanisms of retinal and choroidal neovascularization. Invest. Ophthalmol. Vis. Sci. 35:3974-3979.

33. Favard, C., Ortega, N., Bayard, F., and Plouet, J. 1996. Vascular endothelial growth factor and retinal neovascularisation: a new therapeutic approach for diabetic retinopathy. Diabetes Metab. 22:268-273.

34. Ghiso, N., Rohan, R.M., Amano, S., Garland, R., and Adamis, A.P. 1999. Suppression of hypoxia-associated vascular endothelial growth factor gene expression by nitric oxide via cGMP. Invest. Ophthalmol. Vis. Sci. 40:1033-1039.

35. Shen, B.Q., et al. 1998. Homologous up-regulation of KDR/Flk-1 receptor expression by vascular endothelial growth factor in vitro. J. Biol. Chem. 273:29979-29985

36. Suzuma, K., Takagi, H., Otani, A., Suzuma, I., and Honda, Y. 1998. Increased expression of KDR/Flk-1 (VEGFR-2) in murine model of ischemia-induced retinal neovascularization. Microvasc. Res. 56:183-191.

37. Stone, J., et al. 1996. Roles of vascular endothelial growth factor and astrocyte degeneration in the genesis of retinopathy of prematurity. Invest. Ophthalmol. Vis. Sci. 37:290-299. 